$11-2008$

\title{
On Control System Design for the Conventional Mode of Operation of Vibrational Gyroscopes
}

\author{
Lili Dong \\ Cleveland State University, I.dong34@csuohio.edu \\ Qing Zheng \\ Cleveland State University \\ Zhiqiang Gao

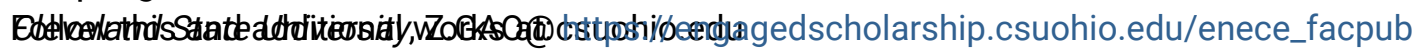 \\ Part of the Electronic Devices and Semiconductor Manufacturing Commons \\ How does access to this work benefit you? Let us know!
}

\section{Publisher's Statement}

(C) 2008 IEEE. Personal use of this material is permitted. Permission from IEEE must be obtained for all other uses, in any current or future media, including reprinting/republishing this material for advertising or promotional purposes, creating new collective works, for resale or redistribution to servers or lists, or reuse of any copyrighted component of this work in other works.

\section{Original Citation}

Lili, D., Qing, Z., \& Zhiqiang, G. (2008). On control system design for the conventional mode of operation of vibrational gyroscopes. IEEE Sensors Journal, 8, 11, 1871-1878.

\section{Repository Citation}

Dong, Lili; Zheng, Qing; and Gao, Zhiqiang, "On Control System Design for the Conventional Mode of Operation of Vibrational Gyroscopes" (2008). Electrical Engineering \& Computer Science Faculty Publications. 93.

https://engagedscholarship.csuohio.edu/enece_facpub/93

This Article is brought to you for free and open access by the Electrical Engineering \& Computer Science Department at EngagedScholarship@CSU. It has been accepted for inclusion in Electrical Engineering \& Computer Science Faculty Publications by an authorized administrator of EngagedScholarship@CSU. For more information, please contact library.es@csuohio.edu. 


\title{
On Control System Design for the Conventional Mode of Operation of Vibrational Gyroscopes
}

\author{
Lili Dong, Graduate Student Member, IEEE, Qing Zheng, and Zhiqiang Gao, Member, IEEE
}

\begin{abstract}
This paper presents a novel control circuitry design for both vibrating axes (drive and sense) of vibrational gyroscopes, and a new sensing method for time-varying rotation rates. The control design is motivated to address the challenges posed by manufacturing imperfection and environment vibrations that are particularly pronounced in microelectromechanical systems (MEMS) gyroscopes. The method of choice is Active Disturbance Rejection Control that, unlike most existing control design methods, does not depend on an accurate model of the plant. The task of control design is simplified when the internal dynamics, such as mechanical cross coupling between the drive and sense axes, and external vibrating forces are estimated and cancelled in real time. In both simulation and hardware tests on a vibrational piezoelectric beam gyroscope, the proposed controller proves to be robust against structural uncertainties; it also facilitates accurate sensing of time-varying rotation rates. The results demonstrate a simple, economic, control solution for compensating the manufacturing imperfections and improving sensing performance of the MEMS gyroscopes.
\end{abstract}

Index Terms-Control circuit, microelectromechanical systems (MEMS), rotation rate estimation, vibrational gyroscopes.

\section{INTRODUCTION}

V ibrational gyroscopes rely on vibrating elements for the purpose of sensing the rotation rate. The quality of such gyroscopes is very much dependent on that of the mechanical vibrations, which are usually regulated by feedback electronics. Therefore, feedback control design plays an important role here. Furthermore, since the vibrational gyroscopes do not use bearings, they can be miniaturized and batch fabricated on silicon or polysilicon, creating MEMS gyroscopes. This makes control design particularly critical. With a long list of advantages concerning size, cost, and power consumption [1], MEMS gyroscopes are nonetheless vulnerable to fabrication imperfection and variation in operating conditions, so much so that the performance and reliability issues have caused difficulty in their applications [2]. Traditional mechanical balancing could reduce the effects of a limited amount of the imperfection. However, it is time consuming, expensive, and difficult to perform on a small-size MEMS gyroscope [3].

From the early 1990s, many researchers have tried to improve the performance of vibrational gyroscopes through better control electronics. Most MEMS gyroscopes operate in a conventional mode [3]-[8], where the movement of the mass along the drive axis is large and the movement along the sense axis is very small. Only a few studies [9], [10] addressed the vibrations of the MEMS gyroscope operating in an adaptive mode, where the movements of a proof mass of the gyroscope along two vibrating axes are equal. In [4]-[7], oscillation controllers were introduced to force the gyroscope to resonance along drive axis. In [8], a Lyapunov-based adaptive controller was designed to control the vibration on both drive and sense axes for the gyroscope, and to estimate a constant rotation rate. A majority of the reported controllers [3]-[10] assume constant rotation rates. In reality, the rotation rate is changing with time. Few papers [11], [12] addressed the problem of estimating time-varying rotation rates. The adaptive controller in [11] is based on previously known displacement and velocity outputs of the MEMS gyroscope. Unfortunately, such velocity states of the two axes are not measurable in practice [9]. The multiple tuning parameters in [11] also make the adaptive controller difficult to implement. The controller in [12] is based on zero coupling terms between two axes of the gyroscope. Actually, the fabrication imperfections cause damping and spring couplings, resulting in measurement errors of the rotation rate. Except for the adaptive controller in [6] where only the drive axis control of the gyroscope is considered, none of the reported controllers [3]-[12] has been validated through hardware implementations.

In this paper, we aim to find better design for the control circuit of both drive and sense axes of the MEMS gyroscope operating in the conventional mode. We propose a solution based on a novel idea known Active Disturbance Rejection Control (ADRC) [13]-[18] that is particularly effective in dealing with a large amount of uncertainties and time-varying parameters, the main challenges pertaining to vibrational gyroscopes. It leads to a solution that is intuitive to understand and easy to use, but, unlike the previous approaches [3]-[10], capable of estimating time-varying rotation rates. Some preliminary results of our research on single-axis vibration control using the ADRC can be found in [19], [20]. In the present paper, we present a complete solution for the vibration control on both axes and the estimation of time-varying rates.

This paper is organized as follows. The dynamics of MEMS gyroscope is discussed in Section II. The controller design is presented in Section III. Simulation results are shown in Section IV, followed by the analog implementation and experi- 
mental results in Section V. Finally, some concluding remarks are given in Section VI.

\section{VibRATIONAL GYROSCOPE DYNAMICS}

The vibrational MEMS gyroscopes can be viewed as a proof mass (inertia element) suspended by elastic structures (springs and dampers) along two orthogonal axes: sense axis ( $Y$ axis) and drive axis ( $X$ axis). For the conventional mode of operation, the proof mass is continuously excited to resonance along the drive axis. Simultaneously, Coriolis acceleration is produced along the sense axis while the sensitive part rotates around a rotation axis (perpendicular to both drive and sense axes) with a rate of $\Omega$. The Coriolis acceleration contains the information of the rotation rate, thus allowing us to determine the rotation rate through sensing the vibration of the sense axis.

For the sake of practicality, we allow for the frequency mismatch between the two axes. The governing equations of the MEMS gyroscope rotating at a time-varying rotation rate are modeled as

$$
\begin{aligned}
& m \ddot{x}+d_{x x} \dot{x}+d_{x y} \dot{y}+\left(k_{x x}-m \Omega^{2}\right) x+k_{x y} y \\
& \quad-2 m \Omega \dot{y}-m \dot{\Omega} y=K u_{x} \\
& m \ddot{y}+d_{y y} \dot{y}+d_{x y} \dot{x}+\left(k_{y y}-m \Omega^{2}\right) y+k_{x y} x \\
& \quad+2 m \Omega \dot{x}+m \dot{\Omega} x=K u_{y}
\end{aligned}
$$

where $x$ and $y$ are the displacement outputs of drive and sense axes, respectively, $\Omega$ is the time-varying rotation rate, $2 m \Omega \dot{x}$ and $2 m \Omega \dot{y}$ represent Coriolis forces (to be used to measure the rotation rate), $m \Omega^{2} x$ and $m \Omega^{2} y$ are centrifugal forces, $m \dot{\Omega} y$ and $m \dot{\Omega} x$ are Euler forces, $k_{x x}$ and $k_{y y}$ are spring constants, $d_{x x}$ and $d_{y y}$ are damping coefficients, $K_{x y}$ and $d_{x y}$ are spring and damping coupling constants between two axes caused by mechanical imperfections, $m$ is the mass of the MEMS gyroscope, $K$ is the controller gain including feed-forward gain and actuator and sensor scale factors, and $u_{x}$ and $u_{y}$ are the control inputs for drive and sense axes, respectively. Since the rotation rate is small compared to the natural frequency of the system and the proof mass is also small (ranging from $10^{-6} \mathrm{~kg}$ through $10^{-10} \mathrm{~kg}$ ), the centrifugal force terms in (1) can be neglected or absorbed as part of the spring terms taken as unknown variations. If we assume the damping coupling is zero [3], [9] and we scale the equations by the mass, we will have the following simplified equations:

$$
\begin{aligned}
& \ddot{x}+2 \zeta_{x} \omega_{x} \dot{x}+\omega_{x}^{2} x+\omega_{x y} y-2 \Omega \dot{y}-\dot{\Omega} y=\frac{k}{m} u_{x} \\
& \ddot{y}+2 \zeta_{y} \omega_{y} \dot{y}+\omega_{y}^{2} y+\omega_{x y} x+2 \Omega \dot{x}+\dot{\Omega} x=\frac{k}{m} u_{y}
\end{aligned}
$$

where $\omega_{x}$ and $\omega_{y}$ are the natural frequencies of drive and sense axes, respectively, $\omega_{x y} y$ and $\omega_{x y} x$ are unknown constant Quadrature errors caused by spring coupling terms between two axes, and $\zeta_{x}$ and $\zeta_{y}$ are damping coefficients of two axes, respectively. Since the rotation rate is an unknown time-varying parameter, the terms $\dot{\Omega} y$ and $\dot{\Omega} x$, which are caused by the Euler forces in the rotating reference frame cannot be neglected. The terms $\dot{\Omega} y$ and $\dot{\Omega} x$ make the most significant difference between (2) and the models described in [3]-[12].
Rotation sensing is achieved by driving the drive axis to resonance, regulating the output of the sense axis, and estimating the Coriolis acceleration of the sense axis. We apply force-rebalancing control strategy onto the sense axis. The basic idea of the force-rebalancing strategy is that, if the amplitude of the output of sense axis is regulated to zero by a feedback controller, then, since $\ddot{y} \cong \dot{y} \cong y \cong 0$, the sense axis model becomes

$$
2 \Omega \dot{x}+\dot{\Omega} x=\frac{k}{m} u_{y}-\omega_{x y} x .
$$

This implies that the rotation rate can be deduced from the sense axis control input $u_{y}$ after the cancellations of Quadrature error.

Therefore, our design objectives are to drive the drive axis to resonance, to force the vibration of sense axis to zero, to cancel out the Quadrature error terms, and to estimate the time-varying rotation rate in the presences of parameter uncertainties and unexpected external disturbances.

\section{PROPOSED SOLUTION}

The existing control design for vibrational gyroscopes are all based rather accurate model of the plant. The reality in practice, however, is quite different. The factors such as the mechanical-thermal noise, the measurement noise, the unknown time-varying rotation rate, and the unknown Quadrature error terms, bring significant modeling errors and structural uncertainties into the system that can not be simply ignored. The mechanical imperfection and environmental variations are another major source of uncertainties in the MEMS gyroscope. Given the nature of the problem, it is imperative that the control system is able to compensate for a large amount of uncertainties, both internal and external. The ADRC is chosen for its inherent disturbance rejection characteristics and its robustness against structural uncertainties. The idea of ADRC is rather straightforward, as shown next.

\section{A. Basic Idea}

Both drive and sense axes of MEMS gyroscopes can be taken as lightly damped second-order systems. They are governed by the Newtonian law of motion. We rewrite (2) as

$$
\begin{aligned}
& \ddot{x}=\left(-2 \zeta_{x} \omega_{x} \dot{x}-\omega_{x}^{2} x-\omega_{x y} y+2 \Omega \dot{y}+\dot{\Omega} y\right)+b_{x} u_{x} \\
& \ddot{y}=\left(-2 \zeta_{y} \omega_{y} \dot{y}-\omega_{y}^{2} y-\omega_{x y} x-2 \Omega \dot{x}-\dot{\Omega} x\right)+b_{y} u_{y}
\end{aligned}
$$

where $b_{x}=b_{y}=K / m$

$$
\begin{aligned}
& f_{x}=-2 \zeta_{x} \omega_{x} \dot{x}-\omega_{x}^{2} x-\omega_{x y} y+2 \Omega \dot{y}+\dot{\Omega} y \\
& f_{y}=-2 \zeta_{y} \omega_{y} \dot{y}-\omega_{y}^{2} y-\omega_{x y} x-2 \Omega \dot{x}-\dot{\Omega} x
\end{aligned}
$$

where $f_{x}$ and $f_{y}$ account for all the other forces excluding the control efforts [13]. The terms $f_{x}$ and $f_{y}$ are also referred to as the generalized disturbance, or disturbance, since they represent both the unknown internal dynamics and the external disturbances of the drive and sense axes, respectively. The couplings between the two axes are taken as disturbances to be rejected as well. Substituting (5) into (4), the system becomes

$$
\begin{aligned}
& \ddot{x}=f_{x}+b_{x} u_{x} \\
& \ddot{y}=f_{y}+b_{y} u_{y} .
\end{aligned}
$$


The basic idea of ADRC is to obtain the estimated $f_{x}$ and $f_{y}$, i.e. $\hat{f}_{x}$ and $\hat{f}_{y}$, and to compensate for them in the control law in real time. Note that the control designs for the drive and sense axes are the same, and they are implemented in parallel. For simplicity, the concept of ADRC is explained with the control of the drive axis only.

Let $\hat{f}_{x}$ be the estimated $f_{x}$ obtained using a state observer. Then

$$
u_{x}=\frac{1}{b_{x}}\left(-\hat{f}_{x}+u_{0}\right)
$$

reduces the drive axis dynamics to a simply double integral plant of the form

$$
\ddot{x}=f_{x}-\hat{f}_{x}+u_{0} \approx u_{0}
$$

which can be easily driven to resonance by using a PD controller. In particular, let

$$
r=A \sin (\omega t)
$$

be the reference signal for the output signal $x$ to track and the tracking error is $e=r-x$, then a simple proportional derivative (PD) controller of the form

$$
u_{0}=k_{p} e+k_{d} \dot{e}
$$

can drive $e$ to zero.

The simplicity of such a design strategy is obviously premised on the accurate, real-time estimation of $f_{x}$, to which we now turn.

\section{B. Extended State Observer}

The effectiveness of ADRC is dependent on the accurate estimation of $f_{x}$. However, no existing state observers can serve for this purpose unless we extend the meaning of "state" to include $f_{x}$, and this gives the name to the extended state observer (ESO), as shown in details in [14]-[16]. Pertaining to the application at hand, let $x_{1}=x, x_{2}=\dot{x}, x_{3}=f_{x}$, and $X=\left[x_{1}, x_{2}, x_{3}\right]^{T}$, an alternative state-space model of the drive axis is obtained as

$$
\begin{aligned}
\dot{X} & =A X+B u_{x}+E h \\
z & =C X
\end{aligned}
$$

where

$A=\left[\begin{array}{lll}0 & 1 & 0 \\ 0 & 0 & 1 \\ 0 & 0 & 0\end{array}\right], B=\left[\begin{array}{c}0 \\ b_{x} \\ 0\end{array}\right], C=\left[\begin{array}{lll}1 & 0 & 0\end{array}\right], E=\left[\begin{array}{l}0 \\ 0 \\ 1\end{array}\right], h=\dot{f}_{x}$.

Based on (11), a state observer is given by

$$
\begin{aligned}
\dot{\hat{X}} & =A \hat{X}+B u_{x}+L(z-\hat{z}) \\
\hat{z} & =C \hat{X}
\end{aligned}
$$

where the estimated state vector is $\hat{X}=\left[\hat{x}_{1}, \hat{x}_{2}, \hat{x}_{3}\right]^{T}$ and the vector of observer gain is $L=\left[l_{1}, l_{2}, l_{3}\right]^{T}$. Note that the key part of (12) is the third state of observer $\hat{x}_{3}$, which is an estimate of $f_{x}$. The characteristic polynomial of the observer is

$$
p(s)=s^{3}+l_{1} s^{2}+l_{2} s+l_{3} .
$$

If the observer gains are selected as $l_{1}=3 \omega_{o}, l_{2}=3 \omega_{o}^{2}, l_{3}=$ $\omega_{o}^{3}$, and $\omega_{o}>0$, the characteristic polynomial becomes

$$
p(s)=\left(s+\omega_{o}\right)^{3} \text {. }
$$

It makes $\omega_{o}$ the only tuning parameter of the observer, which makes the tuning easy in implementation.

Remark: If we take the time-varying rotation rate $\Omega$ as a sinusoidal signal, i.e. $\Omega=A_{\Omega} \sin \left(\omega_{\Omega} t\right)$, and assume that the spring coupling term $\omega_{x y}$ is an unknown constant, then, consider the fact that the velocities and accelerations of $x$ and $y$ axes, the natural frequencies of drive and sense axes, and the frequency $\omega_{\Omega}$ of rotation rate are all bounded, we can conclude that, for the benefits of stability analysis, $\dot{f}_{x}$ is bounded.

\section{Feedback Control Law}

Coupled with the ESO in (12), the control law in (7) becomes

$$
u_{x}=\frac{1}{b_{x}}\left(-\hat{x}_{3}+u_{0}\right)
$$

with $u_{0}$ given in (10). To further simplify the tuning process, the gains of the PD controller is chosen as $k_{p}=\omega_{c}^{2}$ and $k_{d}=2 \omega_{c}$, where $\omega_{c}>0$. Then, (10) becomes

$$
u_{0}=\omega_{c}^{2} e+2 \omega_{c} \dot{e}
$$

Since the velocity of the movement along the drive axis is not measurable, the controller $u_{0}$ built on the observed velocity is given by

$$
u_{0}=\omega_{c}^{2}\left(r-\hat{x}_{1}\right)+2 \omega_{c}\left(\dot{r}-\hat{x}_{2}\right) \text {. }
$$

Note that for better tracking performance, an additional feed-forward term, $\ddot{r}$ was added to (17) in simulation and hardware tests. Assuming accurate estimation of the states, the ideal closed-loop transfer function of controller is

$$
G(s)=\frac{x}{r}=\frac{\omega_{c}^{2}+2 \omega_{c} s}{s^{2}+2 \omega_{c} s+\omega_{c}^{2}} .
$$

From (18), we can see that this is a typical second-order critical-damping control system, where $\omega_{c}$ is the only one tuning parameter for the control law. The details about how to tune the parameters of ADRC can be found in [15].

The ESO in (12) and the control law in (15) and (16) constitute the oscillation controller for the drive axis of the MEMS gyroscope. Under the oscillation control, the output of drive axis $x$ is expected to be driven to the reference $r$, which is a desired sinusoidal signal with constant amplitude and resonant frequency. Since the disturbance $f_{x}$ could be estimated in ESO, the parameter variations and the Quadrature error included in $f_{x}$ will be compensated in the control effort. Another advantage of this oscillation controller is that there are only two tuning parameters $\left(\omega_{c}\right.$ and $\left.\omega_{o}\right)$, making it easy to implement in hardware.

As analyzed in Section II, we apply force-to-rebalance strategy to the sense axis of the gyroscope. The same ADRC algorithm is used to drive the output of sense axis $y$ to be zero $(r=0)$. The remaining task is to estimate the rotation rate. 


\section{Rotation Rate Estimation}

On the sense axis of the MEMS gyroscope, the Coriolis acceleration and Quadrature error terms have a relative phase shift of $90^{\circ}$. Therefore, we can use this characteristic to separate the undesirable Quadrature errors from the useful Coriolis acceleration using the demodulation technique. As the amplitude of $y$ is driven to zero, we have

$$
f_{y}=-\omega_{x y} x-2 \Omega \dot{x}-\dot{\Omega} x
$$

Suppose $x$ has converged to $r$ under the oscillation control on the drive axis, which makes

$$
x \approx A \sin (\omega t)
$$

Note that from [9], the frequency of the rotation rate $f_{\text {rate }}(0$ $\mathrm{Hz} \sim 100 \mathrm{~Hz})$ is far less than the one of $\omega(2 \sim 30 \mathrm{KHZ})$, thus

$$
\begin{aligned}
f_{y} \cdot \cos (\omega t)= & \left(-\omega_{x y} x-2 \Omega \dot{x}-\dot{\Omega} x\right) \cdot \cos (\omega t) \\
= & -\omega_{x y} A \sin (\omega t) \cos (\omega t)-2 \Omega \omega A \cos ^{2}(\omega t) \\
& -\dot{\Omega} A \sin (\omega t) \cos (\omega t) \\
= & -0.5 \omega_{x y} A \sin (2 \omega t)+\Omega \omega A \cos (2 \omega T) \\
& -0.5 \Omega A \sin (2 \omega t)-\Omega \omega A
\end{aligned}
$$

In (21), the high-frequency signals $-0.5 \omega_{x y} A \sin (2 \omega t)$, $-\Omega \omega A \cos (2 \omega t)$, and $-0.5 \dot{\Omega} A \sin (2 \omega t)$ will be filtered out through a low-pass filter (LPF). Therefore, the rotation rate $\Omega$ can be demodulated from the signal $f_{y}$ by the following:

$$
\Omega=F_{\mathrm{LPF}}\left(\frac{-f_{y} \cos (\omega t)}{A \omega}\right)
$$

where $F_{\mathrm{LPF}}($.$) represents a low-pass filter. In (22), the f_{y}$ can be estimated using the ESO. Then, we will have an estimated $\Omega$ as follows:

$$
\hat{\Omega}=F_{\mathrm{LPF}}\left(\frac{-\hat{f}_{y} \cos (\omega t)}{A \omega}\right) .
$$

The transfer function of the LPF used here is

$$
G_{\mathrm{LPF}}(s)=\frac{1}{(\tau s+1)^{2}}
$$

where $\tau$ is the time constant of the filter.

\section{Simulation Results}

We simulated the ADRC solution on the model of a piezoelectrically driven vibrational beam gyroscope (Fig. 1), which is an alternative to the MEMS gyroscope for experimental use. The vibrational beam gyroscope consists of a $20 \mathrm{~mm}$-long steal beam and four piezoelectric strips, which are attached to each side of the beam functioning as actuators and sensors [21]. The beam is driven to resonance through piezoelectric actuators. The deformation (or displacement) of the beam is transformed to voltage and output from the piezoelectric sensors. As the gyroscope is rotating, we can sense the deformation to determine the

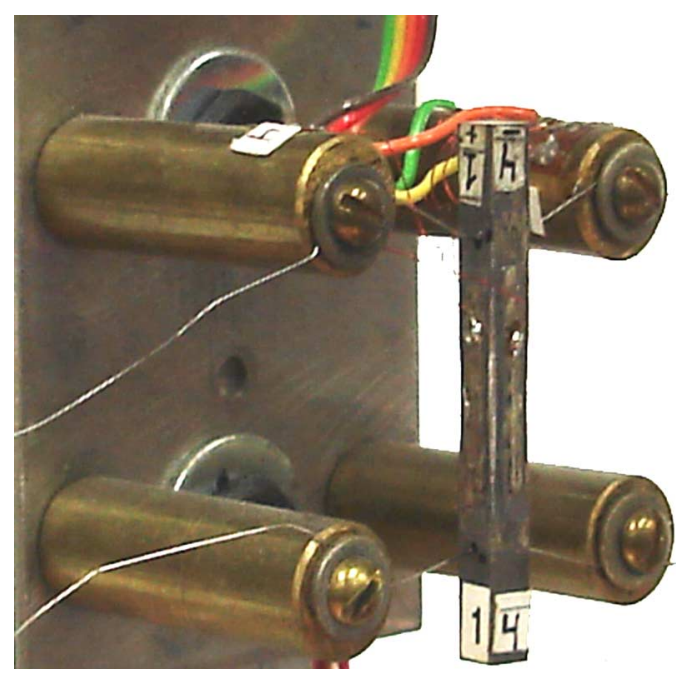

Fig. 1. Photo of a vibrational beam gyroscope.

rotation rate. The voltage output of the actuator is proportional to its displacement. The maximum amplitude of the output of the gyroscope is $100 \mathrm{mv}$, and the corresponding displacement is approximately $10^{-6} \mathrm{~m}$. So, we choose $100 \mathrm{mv}$ as the desired amplitude. We use $A=0.1$ in simulation units to represent this. The natural frequencies and damping coefficients of the gyroscope are $\omega_{x}=63881.1 \mathrm{rad} / \mathrm{s}, \omega_{y}=63681.1 \mathrm{rad} / \mathrm{s}$, and $\zeta_{x} \approx \zeta_{y}=0.0005 \mathrm{~N} \cdot \mathrm{s} / \mathrm{m}$. The desired frequency of drive axis is $\omega=65973.4 \mathrm{rad} / \mathrm{s}(f=10.17 \mathrm{KHz})$, which is approximately equal to its natural frequency. Then, the reference signal $r=0.1 \sin (65973.4 t)$. We assume the magnitude of Quadrature error term is $0.1 \%$ of natural frequency according to [9]. The rotation rate is $\Omega=0.1 \sin \left(2 \pi f_{\text {rate }} t\right) \mathrm{rad} / \mathrm{s}$. The coefficient of controller $b=K / m=271780942.56$ [6]. For the ADRC and ESO, we choose a controller gain $\omega_{c}$ as $5 \times 10^{4}$ and an observer gain $\omega_{o}$ as $25 \times 10^{4}$. The oscillation controller is represented by

$$
u_{x}=-\frac{1}{b_{x}} \hat{x}_{3}+\frac{\omega_{c}^{2}}{b_{x}}\left(r-\hat{x}_{1}\right)+\frac{2 \omega_{c}}{b_{x}}\left(\dot{r}-\hat{x}_{2}\right)
$$

where $\omega_{c}$ and $\omega_{c}^{2}$ are divided by the coefficient $b$, greatly reducing the large magnitudes of the controller gains. We use the same controller for the sense axis operating under force-to-rebalance mode, where $r=0$. Therefore

$$
u_{y}=-\frac{1}{b_{y}} \hat{y}_{3}-\frac{\omega_{c}^{2}}{b_{y}} \hat{y}_{1}-\frac{2 \omega_{c}}{b_{y}} \hat{y}_{2}
$$

where $\hat{y}_{3}$ is the estimated $f_{y}, \hat{y}_{1}$ is the estimated $y$, and $\hat{y}_{2}$ is the estimated $\dot{y}$. Fig. 2 shows the excellent tracking of real output of drive axis $x$ to the reference signal $r$. Fig. 2(a) is a wide image of both $r$ and $x$. Fig. 2(b) is a close-up view of them. Fig. 3 shows the output $y$ of sense axis, which is driven to almost zero by the ADRC. Fig. 3(a) is a wide image of $y$. Fig. 3(b) is a close-up view of $y$. Fig. 4 shows the estimation of a constant rotation rate with the amplitude of 0.1 . Fig. 5 shows the estimation of a time-varying rotation rate $(\Omega)$ with the rate frequency of $50 \mathrm{~Hz}$. Fig. 5(a) is a wide image of both actual $\Omega$ and the estimated $\Omega$. 


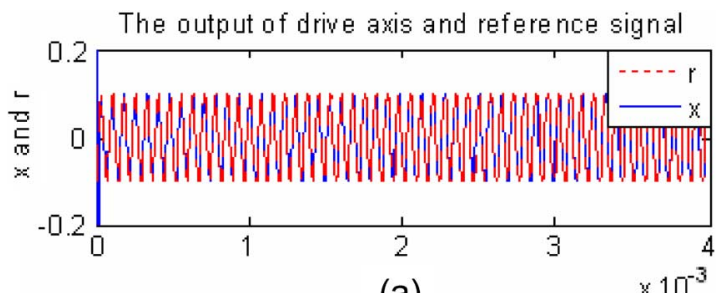

(a)

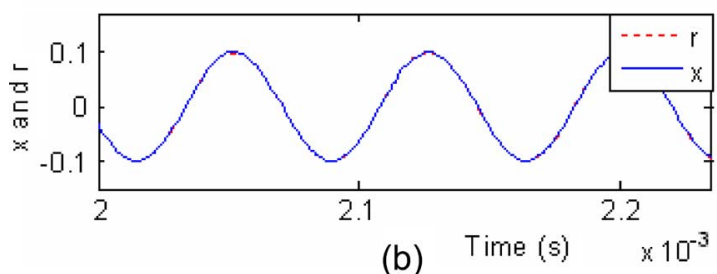

Fig. 2. The real output of the drive axis and the reference signal.

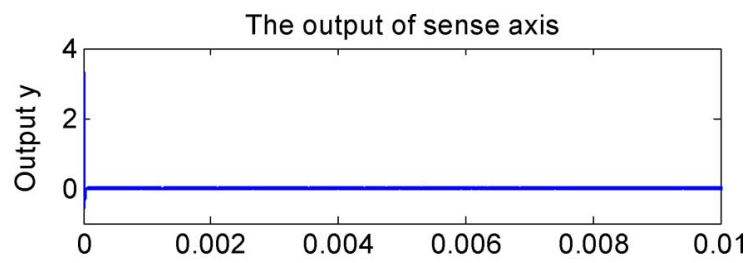

(a)

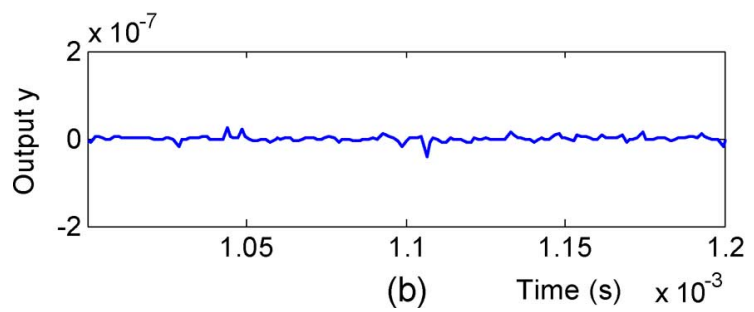

Fig. 3. The output of the sense axis.

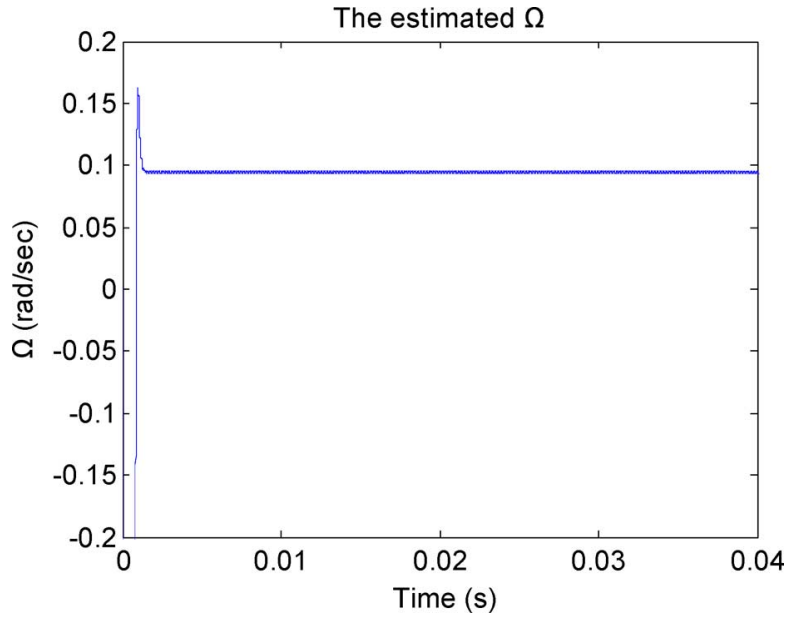

Fig. 4. The rotation rate estimation as $\Omega=0.1$.

Fig. 5(b) is a close-up view of them. From Figs. 4 and 5, we can see that the ADRC can accurately estimate both time-varying and time-invariant rotation rates. The estimated rotation rates match the actual rotation rates very well. Since the approximation of rotation rate is dependent on the accurate estimations of

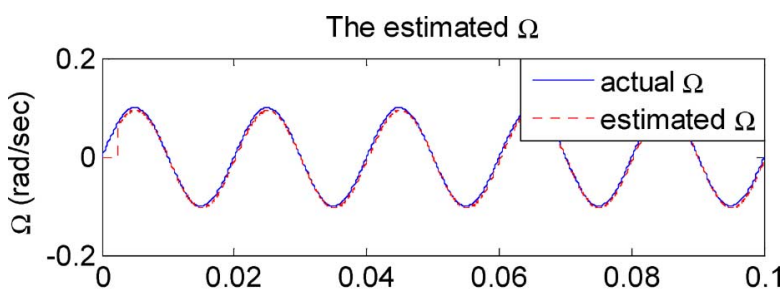

(a)

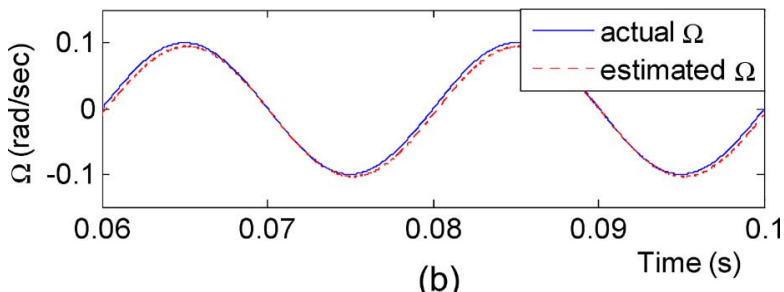

Fig. 5. The rotation rate estimation at $f_{\text {rate }}=50 \mathrm{~Hz}$.
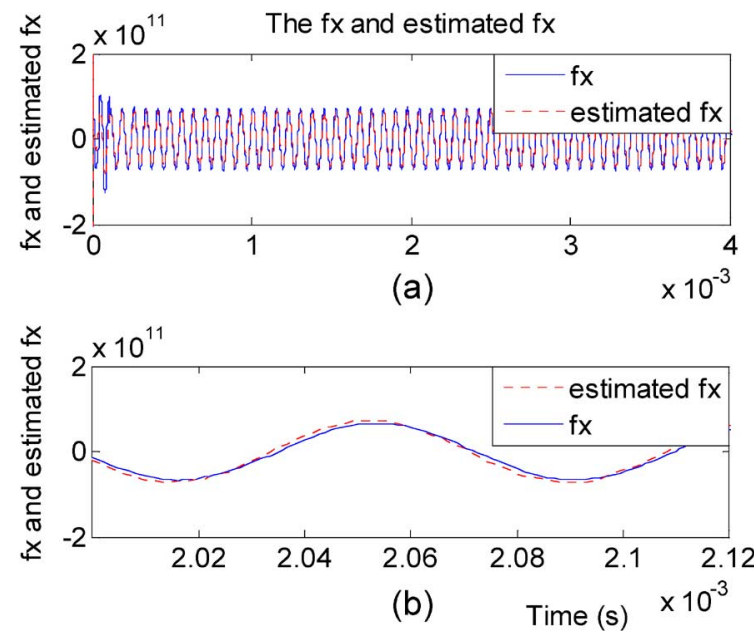

Fig. 6. The $f_{x}$ and estimated $f_{x}\left(\hat{f}_{x}\right)$.
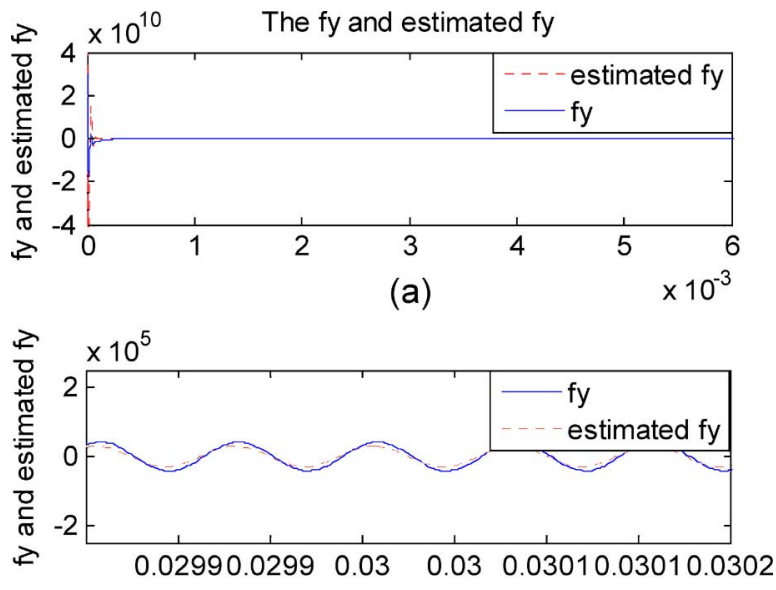

(b)

Time (s)

Fig. 7. The $f_{y}$ and estimated $f_{y}\left(\hat{f}_{y}\right)$.

$f_{x}$ and $f_{y}$, the estimated $f_{x}$ and $f_{y}$ versus time along with real states versus time are given in Figs. 6 and 7. The stimulation results verified the theoretical feasibility of the ADRC. 


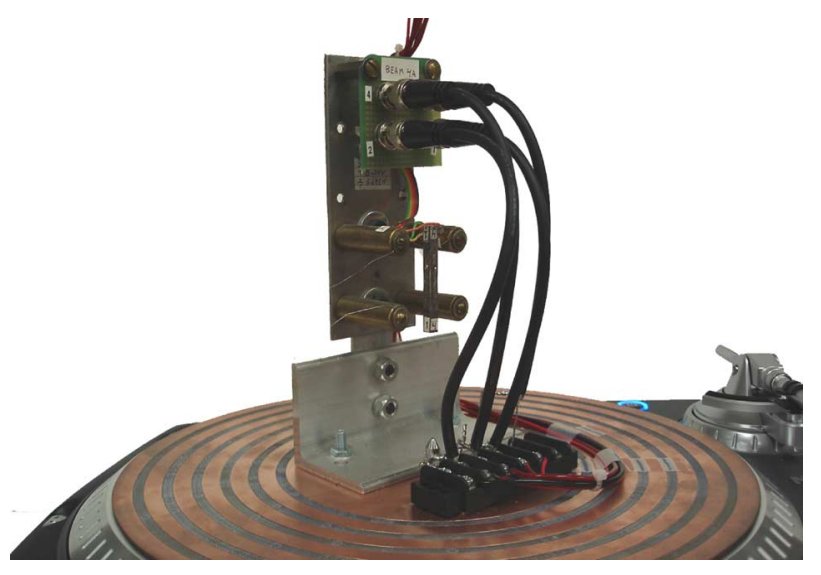

Fig. 8. Experimental setup photo of vibrational beam gyroscope and rate table.

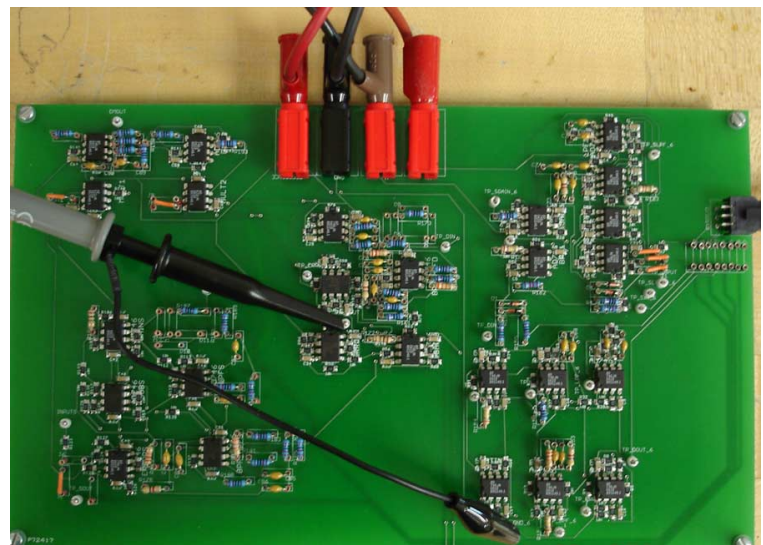

Fig. 9. The PCB for the analog circuit implementation of the ADRC.

\section{ANALOG IMPLEMENTATION AND EXPERIMENTAL RESULTS}

In this section, we will testify the practical feasibility of the ADRC through a hardware implementation. The ADRC can be implemented in two different ways: analog and digital. Field programmable gate array (FPGA) and DSP-base digital implementations of the ADRC have been introduced in [18], [22]. This paper shows how the analog implementation of the ADRC can be accomplished for both axes of the vibrational gyroscope. The analog implementation is fast and inexpensive, making it a practical solution to the electronic design problem of the current inertial rate sensor industry where the cost and responding speed are of great concern. The experimental setup of the gyroscope and a rotation rate table is shown in Fig. 8. The rate table, where the vibrational beam gyroscope is mounted, is rotating at a constant rate. The output signals of the gyroscope are sent to the control electronic circuit board through suspended wires, which are designed to avoid strangling during the rotating. The ADRC is constructed using the circuits consisting of only discrete active or passive components and the circuits are integrated into a printed circuit board (PCB), as shown in Fig. 9. The block diagram of the hardware implementation of the control system for the gyroscope is shown in Fig. 10. The PCB consists of a signal generator circuit, the ADRC (including ESO), the modulator/demodulator circuit for rate estimation, and a saturator. The signal generator is used to produce the reference signal for

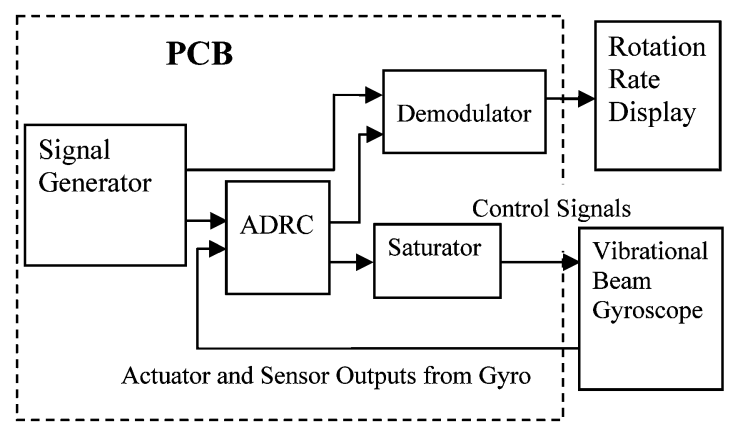

Fig. 10. The control system for the vibrational gyroscope.

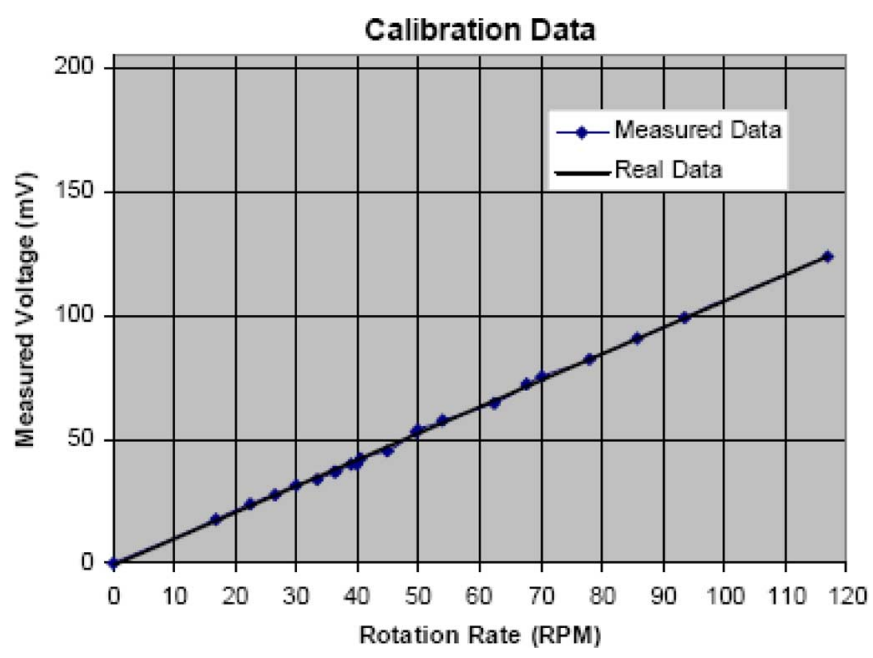

Fig. 11. Calibration data between the rotation rates of the rate table and the output voltages.

the drive axis and to produce the carrier signal for the modulation/demodulation circuit. A saturator is used to limit the magnitudes of control signals. The rotation rate information (voltage levels) is output from demodulation circuit, calibrated, and compared with real rotation rates of the rate table.

The measured and the real rotation rates of the rate table verses the output voltages of the demodulation circuit are shown in Fig. 11, where we can see that the measured rotation rates match the real rates very well. In the experiment, we choose $100 \mathrm{mv}$ as the desired amplitude of the reference signal. The other parameters of the beam gyroscope are same as the ones in simulation. They are subject to the variations of $\pm 10 \%$ the original values in practical situation. The whole output of drive axis is shown in Fig. 12. The output of sense axis, which is driven to almost zero, is shown in Fig. 13. The measurements of the rotation rates at 0, 16.7, 33.3, and 50 RPM are shown in Fig. 14.

From the experimental results, we can see that the analog implementation of the ADRC produces a very fast response, as shown in Fig. 12, where the amplitude of the output of the drive axis reaches the desired value $(0.1 \mathrm{v})$ within $0.14 \mathrm{~ms}$ only. It generally takes 60 microseconds for the frequency of the output of the signal generator to reach the resonant frequency of the drive axis. Therefore, the amplitude of the output response of the drive axis increases dramatically after 60 microseconds in the Fig. 12. The experimental results verified the effectiveness of the ADRC in a real-world situation. 


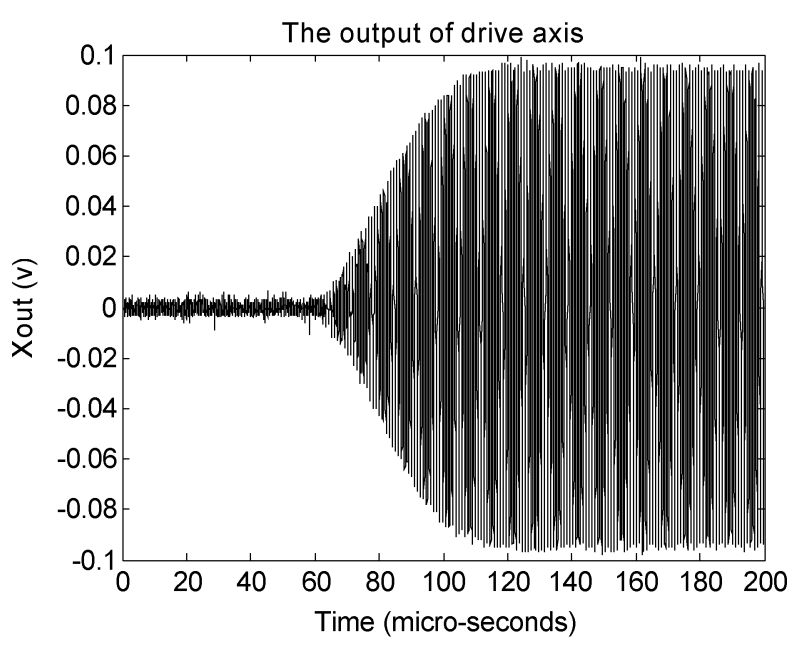

Fig. 12. The output of drive axis.

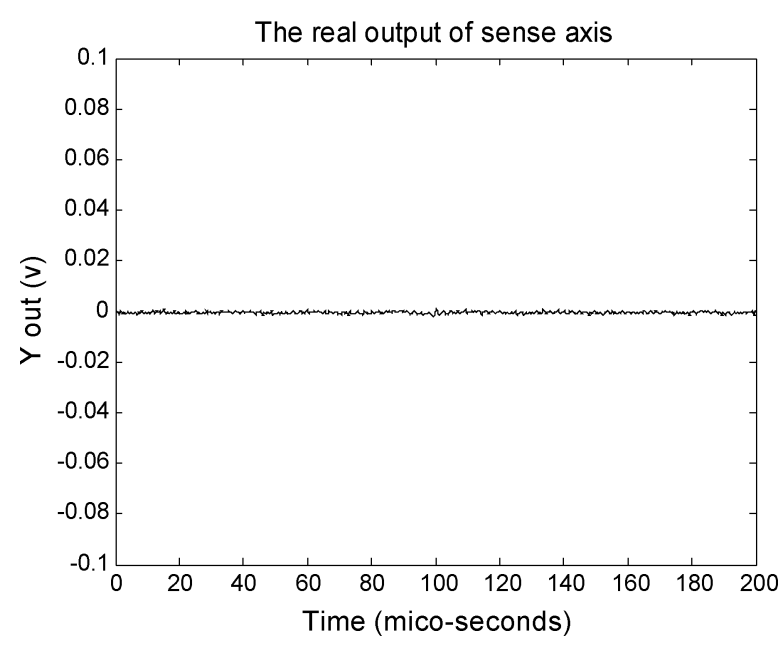

Fig. 13. The output of sense axis.

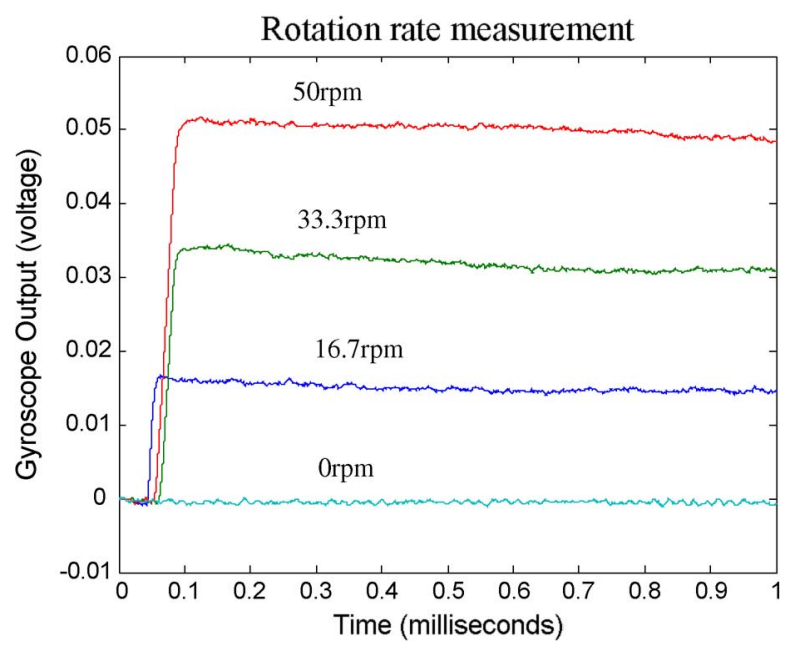

Fig. 14. Approximations of constant rotation rates.

\section{CONCLUDING REMARKS}

A high-performance and low-cost control solution is proposed in this paper for vibrational gyroscopes, based on the active disturbance rejection concept. By a detailed examination of the gyroscope dynamics and the design objectives, we found this concept offers an almost idea fit for the problem at hand, where the uncertainties due to manufacturing imperfection and unknown disturbances from the operating environment are actively estimated and cancelled, leaving the designer with a simple, double integral, plant to deal with. Both simulation and hardware test results strongly substantiate such a simple, effective, approach to a complex control problem. As a direct consequence of the new control design, the rotation rate measurement becomes rather straightforward and consistently accurate. Furthermore, such a solution could very well be suitable for other sensors including MEMS micro-accelerometers since they share a similar dynamic structure.

\section{ACKNOWLEDGMENT}

The authors gratefully acknowledge the University of Alabama for contributing the beam gyroscope for our experimental use, and D. Avanesian and A. Roberts for building the circuits and acquiring experimental data.

\section{REFERENCES}

[1] N. Barbour and G. Schemidt, "Inertial sensor technology trends," IEEE Sensors J., vol. 1, no. 4, pp. 332-339, Dec. 2001.

[2] Y. Yazdi, F. Ayazi, and K. Najafi, "Micromachined inertial sensors," Proc. IEEE, vol. 86, no. 8, pp. 1640-1659, Aug. 1998.

[3] S. Park and R. Horowitz, "Adaptive control for the conventional mode of operation of MEMS gyroscopes," J. Microelectromech. Syst., vol. 12, no. 1, pp. 101-108, Feb. 2003.

[4] C. Acar and A. M. Shkel, "An approach for increasing drive-mode bandwidth of MEMS vibratory gyroscopes," J. Microelectromech. Syst., vol. 14, no. 3, pp. 520-528, 2005.

[5] R. T. M'Closkey and A. Vakakis, "Analysis of a micromsensor automatic gain control loop," in Proc. Amer. Control Conf., San Diego, CA, Jun. 1999, vol. 5, pp. 3307-3311.

[6] R. P. Leland, "Adaptive mode uning for vibrational gyroscopes," IEEE Trans. Control Syst. Technol., vol. 11, no. 2, pp. 242-247, Mar. 2003.

[7] R. P. Leland, Y. Lipkin, and A. Highsmith, "Adaptive oscillator control for a vibrational gyroscope," in Proc. Amer. Control Conf., Denver, CO, June 2003, pp. 3347-3352.

[8] R. Leland, "Adaptive control of a MEMS gyroscope using Lyapunov methods," IEEE Trans. Control Syst. Technol., vol. 14, no. 2, pp. 278-283, Mar. 2006.

[9] S. Park, "Adaptive Control strategies for MEMS gyroscopes," Ph.D. dissertation, Dept. Mech. Eng., Univ. California, Berkeley, CA, 2000.

[10] J. D. John and T. Vinay, "Novel concept of a single-mass adaptively controlled triaxial angular rate sensor," IEEE Sensors J., vol. 6, no. 3, pp. 588-595, Jun. 2006.

[11] L. Dong and R. P. Leland, "The adaptive control System of a MEMS gyroscope with time-varying rotation rate," in Proc. Amer. Control Conf., Portland, OR, Jun. 2005, vol. 5, pp. 3592-3597.

[12] M. Salah, M. McIntyre, D. Dawson, and J. Wagner, "Time-varying angular rate sensing for a MEMS Z-axis gyroscope," in Proc. 45th IEEE Conf. Decision and Control, Dec. 2006, pp. 2165-2170.

[13] D. Sun, "Comments on active disturbance rejection control," IEEE Trans. Ind. Electron., vol. 54, no. 6, pp. 3428-3429, Dec. 2007.

[14] Z. Gao, "Active disturbance rejection control: A paradigm shift in feedback control system design," in Proc. Amer. Control Conf., Minneapolis, MN, Jun. 2006, pp. 2309-2405.

[15] Z. Gao, "Scaling and bandwidth-parameterization based controller tuning," in Proc. Amer. Control Conf., Denver, CO, Jun. 2003, vol. 6, pp. 4989-4996.

[16] Z. Gao, Y. Huang, and J. Han, "A novel motion control design approach based on active disturbance rejection," in Proc. 40th IEEE Conf. Decision and Control, Orlando, FL, Dec. 2001, vol. 5, pp. 4578-4585.

[17] Z. Gao, T. Thomas, and J. Dawson, "A stable self-tuning fuzzy logic control system for industrial temperature control problems," IEEE Trans. Ind. Appl., vol. 38, no. 2, pp. 414-424, Apr. 2002.

[18] B. Sun and Z. Gao, "A DSP-based active disturbance rejection control design for a $1 \mathrm{KW}$ H-bridge DC-DC power converter," IEEE Trans. Ind. Electron., vol. 52, no. 5, pp. 127-1277, Oct. 2005. 
[19] L. Dong, Q. Zheng, D. Avanesian, and Z. Gao, "The design and implementation of driving mode control for vibrational gyroscopes," in Proc. Amer. Control Conf., Seattle, WA, Jun. 2008, pp. 4419-4424.

[20] Q. Zheng, L. Dong, and Z. Gao, "Control and time-varying rotation rate estimation of vibrational MEMS gyroscopes," in Proc. 2007 IEEE Multi-Conf. Syst. Control, Singapore, Oct. 2007, pp. 118-123.

[21] A. Katz and A. Highsmith, "The optimal size of a resonant vibrating beam gyroscope," J. Dynamic Syst.s, Measure. Control, vol. 123, pp. 49-53, Mar. 2001.

[22] Z. Ping, "A hardware-reconfigurable control platform for space power management and distribution systems," Doctorial dissertation, Dept. Elect. Comput. Eng., Cleveland State Univ., Cleveland, OH, 2005. 